\title{
Trastornos Alimentarios y Funcionamiento Familiar Percibido en una Muestra de Estudiantes Secundarias de la Comuna de Concepción, Chile
}

\section{Eating Disorders and Perceived Family Functioning in a Sample of High School Students of Concepción, Chile}

\author{
Claudia Cruzat \\ Patricio Ramírez \\ Roberto Melipillán \\ Paula Marzolo \\ Universidad del Desarrollo
}

\begin{abstract}
Los trastornos alimentarios son patologías altamente frecuentes en adolescentes, con relativo mal pronóstico y dificultades en su abordaje. La literatura señala que las familias tienden a ser más disfuncionales que los controles y la calidad del funcionamiento familiar juega un rol importante en su curso. Esta investigación busca establecer relaciones entre síntomas psicológicos asociados a un trastorno alimentario en estudiantes secundarias de la comuna de Concepción y el tipo de funcionamiento familiar percibido. Se aplicó el EDI-2 y TUSU a una muestra de 296 adolescentes. Los principales hallazgos señalan que la relación esperada se repite en población no clínica y se destaca la importancia de la comunicación con el padre, elemento que debiera ser profundizado en nuevas investigaciones.
\end{abstract}

Palabras claves: trastornos alimentarios, funcionamiento familiar, adolescencia.

\begin{abstract}
Eating disorders are highly prevalent in adolescence, have relative bad prognosis and present difficulties for their treatment. Research indicates that families from adolescents suffering from eating disorders tend to be more dysfunctional than control families and that the quality of the family's functioning plays a key role in the course of the disease. The goal of this study was to identify relations between psychological symptoms associated with eating disorders and perceived family functioning among high school students. A sample of 296 adolescents from Concepción responded to the EDI2 and TUSU. The main findings replicated the hypothesized relationship in a non-clinical population and emphasized the importance of father-adolescent communication. Future research should further study the role of father-adolescent communication in the emergence and maintenance of eating disorders.
\end{abstract}

Keywords: eating disorders, familiar functioning, adolescence.

Por distintas razones, los trastornos alimentarios son enfermedades emergentes que han despertado en los últimos años un enorme interés en medios sociales y sanitarios. La incidencia de anorexia nerviosa parece haber aumentado en las décadas recientes, tanto en nuestro país como en países extranjeros (Maturana, 2003). Se trata de enfermedades

Claudia Verónica Cruzat Mandich, Facultad de Psicología. Universidad del Desarrollo. Chile.

Patricio Ramírez Azócar, Facultad de Psicología. Universidad del Desarrollo. Chile.

Roberto Melipillán Araneda, Facultad de Psicología. Universidad del Desarrollo. Chile.

Paula Marzolo Uribe, Facultad de Psicología. Universidad del Desarrollo. Chile.

La correspondencia relativa a este artículo debe ser dirigida a Claudia Verónica Cruzat, Facultad de Psicología, Universidad del Desarrollo, Ainavillo 456, Concepción, Chile. E-mail: ccruzat@udd.cl

Esta investigación fue realizada gracias al apoyo financiero de la Dirección de Investigación de la Universidad del Desarrollo. psicosomáticas que figuran entre los problemas de salud crónicos más frecuentes en los adolescentes, y cuya letalidad es la más alta de las detectadas por trastornos psiquiátricos.

La revisión de estudios realizados en varios países europeos, asiáticos y Estados Unidos por van Hoeken, Seidell y Hoek (2003) indica que la prevalencia promedio para las adolescentes es de $0.3 \%$ para la anorexia nerviosa y $1 \%$ para la bulimia nerviosa. La incidencia total es al menos 8 por cada 100 mil personas al año para la anorexia nerviosa y 12 por cada 100 mil personas al año para la bulimia. Además, la incidencia de anorexia nerviosa ha aumentado en los últimos 50 años, particularmente en mujeres entre 10 y 24 años de edad.

Además, la prevalencia estimada para trastorno por "atracón" (Binge Eating Disorder) es de al menos un 1\% (Hoek \& Van Hoeken, 2003).

Estudios longitudinales y de seguimiento a largo plazo establecen un mal pronóstico de curación para la anorexia, con resultados $2 / 3$ siguen siendo 
restrictivos, 1/3 mantienen bajo peso, 50\% siguen con temor a engordar (Maturana, 2003).

En un estudio transcultural de Steinhausen, Boyadjieva, Grigoroiu-Serbanescu, Seidel y Winkler Metzke (2000) se realizó un seguimiento a 138 pacientes adolescentes fundamentalmente anoréxicas en un periodo de 5 años luego de su primera admisión, encontrándose que en promedio los pacientes habían ocupado el $25 \%$ del tiempo de seguimiento en tratamientos con hospitalización o ambulatorios. En tanto que la mitad de ellos requirieron una segunda hospitalización y un cuarto, una tercera. Un $68 \%$ del total de la muestra ya no cumplía los criterios para un trastorno de alimentación luego del seguimiento.

Por otra parte, el suicidio es la mayor causa de muerte en personas con anorexia nerviosa refutando la idea de que la inanición es la primera causa de muerte. El porcentaje de suicidio de adolescentes con trastornos de alimentación va desde el 1.8 al 7.3\% (Pompili, Mancinelli, Girardi, Ruberto \& Tatarelli, 2004).

Dado este escenario, surge la relevancia de comprender estos trastornos desde distintas perspectivas, para abordarlo preventiva y/o curativamente de manera más efectiva. Una de las maneras de entenderlo es desde el punto de vista de la familia. Es así como en ocasiones nos enfrentamos a las dicotomías, fundamentalmente en el ámbito clínico, entendiendo a la familia como "causante" versus la familia que se "adapta" a la enfermedad o que "padece" sus consecuencias, la familia como culpable o la familia como víctima, la familia como impedimento o como recurso para el tratamiento (Carrasco, 2004).

Numerosas investigaciones han demostrado la relación entre ciertos estilos de funcionamiento familiar con la presencia de un trastorno de la alimentación (anorexia o bulimia), por lo que el presente estudio busca indagar en dicha relación pero en población no clínica, es decir, adolescentes aparentemente "sanas".

\section{Definición de los Trastornos Alimentarios}

Los trastornos alimentarios son alteraciones de las conductas relacionadas con la ingesta, consecuencia de los dramáticos esfuerzos por controlar el peso y la silueta. Los tipos más comunes de trastorno de alimentación, que aparecen tanto en adolescentes como en adultos, son la anorexia nerviosa y bulimia nerviosa (García-Palacios, Rivero \& Botella, 2004).
La característica esencial de la anorexia nerviosa consiste en el rechazo a mantener el peso corporal mínimo normal, en un miedo intenso a ganar peso y en una alteración significativa de la percepción de la forma o tamaño del cuerpo (American Psychiatric Association [APA], 2002).

En la anorexia nerviosa, lo que la define no es una auténtica pérdida o falta de apetito, sino el deseo irrefrenable de alcanzar la delgadez a toda costa (Behar, 2004). Es habitual que esta alteración se inicie por recibir alguna crítica directa o indirecta en relación a su peso (Perpiñá, 1995). Los investigadores han descubierto que los anoréxicos en realidad sienten hambre. Experimentan sensaciones cognoscitivas de hambre, junto con una fuerte preocupación por la comida (Sarason \& Sarason, 1996).

Otra característica es la distorsión de la imagen corporal, a pesar que en las últimas revisiones se ha puesto de manifiesto que no es una característica patognomónica exclusiva de la anorexia nerviosa (Perpiña \& Baños, 1990; Rosen \& Srebnik, 1990 citado en Perpiñá, 1995), y no siempre se manifiesta en estos términos.

Por su parte, la bulimia nerviosa se caracteriza por episodios cíclicos y recurrentes de voracidad (atracones) seguidos por conductas compensatorias inadecuadas como el vómito autoinducido, el abuso de laxantes y diuréticos, el ayuno drástico y/o el ejercicios excesivos (American Psychiatric Association [APA], 2002). La paciente está consciente de que su patrón alimentario no es normal, que escapa de su control, por lo que se angustia enormemente y siente posteriormente gran culpa, autodesprecio, vergüenza y depresión (Behar, 2004). El atracón finaliza debido al malestar físico que produce o por factores externos. Al igual que en las anoréxicas, siempre está presente el temor a engordar y la preocupación por la imagen corporal, lo que produce gran ansiedad (Marín, 2002).

\section{Factores Familiares en el Desarrollo y Manten- ción de los Trastornos Alimentarios}

El interés en el rol de los factores familiares en el desarrollo y mantención de los trastornos alimentarios data de 1873 con la descripción de la Anorexia Nerviosa de Lasègue (Fornari, Wlodarczyk-Bisaga, Matthews, Sandberg, Mandel \& Katz, 1999). Aproximaciones más sistemáticas de investigación de variables familiares en la etiología de los trastornos alimentarios fueron iniciados por Minuchin et al. (1978) y por Selvini-Palazzoli y la 
escuela de Milán de Terapia Familiar (1988 citados en Fornari et al., 1999; Carrasco, 2004).

Conceptualizaciones tempranas de anorexia nerviosa en adolescentes proponían modelos de familias "psicosomáticas" (Minuchin, Rosman \& Baker, 1978 citado en Cook-Darzens, Doyen, Falissard \& Mouren, 2005) o "anorexígenas" (Selvini-Palazzoli, 1978 citado en Cook-Darzens et al., 2005), sugiriendo que patrones familiares específicos o disfunciones contribuían al desarrollo, mantenimiento o perpetuación del trastorno. Basados casi exclusivamente en observaciones clínicas, varias de estas teorías dominaron en los años 70 y 80 , convergiendo en una descripción de la familia anoréxica como típicamente fusionada, rígida y dedicada al autosacrificio y lealtad grupal (Stierlin \& Weber 1989 citado en Cook-Darzens et al., 2005).

Algunos estudios han identificado ciertos estilos de funcionamiento en familias con individuos con trastorno de la alimentación e indican que las interacciones en estas familias difieren de aquellas sin miembros con trastornos alimentarios (Kog \& Vandereycken, 1989; Humphrey, 1989 citados en Fornari et al., 1999).

Uno de los autores más influyentes de tal teoría, Salvador Minuchin, desarrolló un modelo estructural familiar (Minuchin et al., 1978 citado en Carrasco, 2004; Cook-Darzens et al., 2005; Vidovic, Juresa, Begovac, Mahnik, \& Tocilj, 2005) que identificaba cinco características predominantes de interacciones que mantienen la "somatización": (a) aglutinamiento, una extrema forma de proximidad e intensidad en las interacciones familiares; (b) sobreprotección, reflejado en el alto grado de preocupación que los miembros de la familia tienen del bienestar de los otros; (c) rigidez, caracterizado por una necesidad de mantener el status quo; (d) evitación de conflicto; (e) involucración de la hija en los conflictos parentales (maritales) a través de triangulación.

Al interior de la comunidad de científicos que ha estudiado la asociación entre un tipo de funcionamiento familiar y la presencia de trastornos de la alimentación, existen puntos de vista controversiales. Pese a los resultados inconsistentes, han surgido dos grandes hallazgos de la acumulación de datos: (a) mientras parece no haber un patrón específico de funcionamiento familiar asociado a trastornos de alimentación, estas familias generalmente tienden a ser más disfuncionales que las familias control y (b) la calidad del funcionamiento familiar juega un rol importante en el curso y resultado del trastorno (Cook-Darzens et al., 2005).
Los hallazgos de Waller, Calam y Slade (1988) indican que los sujetos con trastornos alimentarios perciben a sus familias como significativamente menos adaptables, menos cohesionadas y más pobremente comunicativas comparadas con personas control sin trastornos alimentarios.

Kagan y Squires (1985 citados en Wisotsky et al., 2003) encontraron que las percepciones de funcionamiento familiar en una muestra de estudiantes no clínica no estaba relacionado con la conducta de dieta, aunque el comer compulsivo estaba asociado con la carencia de cohesión familiar, una característica encontrada a menudo en sujetos con bulimia.

Otros estudios también han examinado el ambiente familiar en estudiantes universitarios no clínicos y encontraron que, en la medida que la disfunción familiar se incrementa, aumenta también el reporte de trastornos de conducta alimentaria (Lundholm \& Waters, 1991 citados en Wisotsky et al., 2003). Así, los trastornos de la conducta alimentaria podrían ser entendidos como un síntoma de la disfunción familiar.

Al comparar la percepción del funcionamiento familiar que tienen hijas con trastornos de la alimentación con la percepción de sus padres, Dancyger, Fornari, Scionti, Wisotsky y Sunday (2005) encontraron diferencias estadísticamente significativas entre las pacientes y sus padres. Las madres consideraron que el funcionamiento familiar era significativamente saludable y poco caótico, en cambio sus hijas no coincidieron con tal reporte. Esta diferencia de percepción, según los autores, puede contribuir a la continuación de los patrones de funcionamiento familiar disfuncionales y así, a mantener la presencia de los trastornos de la alimentación o a impactar negativamente en el curso del tratamiento.

Otros estudios han examinado la relación entre sintomatología de trastorno alimentario y rasgos medidos a través del EDI y funcionamiento familiar percibido. Lundholm y Waters (1991 citados en Wisotsky et al., 2003) reportaron que las subescalas del EDI que miden perfeccionismo (excesiva expectativa personal) y Conciencia Interoceptiva (carencia de habilidad para reconocer emociones o sensaciones de hambre o saciedad) se diferenciaban significativamente entre los tipos de familia percibido. Estos autores señalan que en la medida que la disfunción familiar aumenta, el reporte de trastornos de la conducta alimentaria también aumenta, por lo que concuerda con lo señalado por Minuchin (1978) en que las dinámicas familiares de 
aglutinamiento, sobreprotección y rigidez juegan un papel importante en el desarrollo y/o mantención de dichos trastornos.

En el estudio de Wisotsky et al. (2003) con 65 pacientes entre 12 y 27 años se buscaba examinar la relación entre los niveles de funcionamiento familiar percibido y trastornos de alimentación en tres grupos distintos de trastornos, utilizando la escala FACES-II de Olson et al. (1982) y la escala EDI-2. Un hallazgo importante de esta investigación, y que apoya los postulados de la teoría, es que a medida que la disfunción familiar crece, la gravedad de los trastornos de alimentación también lo hace.

Un estudio realizado en México con 200 estudiantes de primer año de dos preparatorias, una pública y otra privada, tuvo como objetivo la identificación de la incidencia de sintomatología de trastornos alimenticios, junto con la indagación acerca del nivel de funcionalidad familiar. Los investigadores aplicaron un cuestionario desarrollado de acuerdo a los propósitos del estudio, encontrando que una de cada cuatro estudiantes padece síntomas relacionados con trastornos alimenticios y que factores familiares como la percepción de una madre perfeccionista y un padre periférico, dificultan la comunicación interna. Además, encontraron un lazo excesivo de lealtad y dependencia familiar en esta misma parte de la muestra (Hernández, 2006).

\section{Diferencias en el Funcionamiento Familiar Percibido Entre los Subtipos de Trastornos Alimentarios}

La administración de inventarios estandarizados a padres de sujetos con anorexia ha producido pequeñas diferencias respecto de los controles normales (Crisp, Harding \& McGuinness, 1974; Garfinkel et al., 1983; Strober, Salkin, Burroughs \& Morrell, 1982, citados en Vitousek \& Manke, 1994), observándose ciertos indicadores de hostilidad, impulsividad y descontrol en los padres de anoréxicasbulímicas (Strober et al., 1982 citado en Vitousek \& Manke, 1994) y por otra parte, orientación al logro en los padres de anoréxicas restrictivas (Crisp et al., 1974; Garfinkel et al., 1983 citados en Vitousek \& Manke, 1994).

Por otra parte, se ha encontrado que las interacciones familiares en sujetos con síntomas de anorexia y bulimia difieren, en el sentido de que sujetos con anorexia describen a sus familias como cohesivas y organizadas con una tolerancia al conflicto más baja (Goldstein, 1981; Garfinkel, Garner \& Rose, 1983 citados en Fornari et al., 1999; Vidovic, Juresa, Begovac, Mahnik \& Tocilj, 2005), opuesto a los sujetos con bulimia quienes reportan a sus familias como significativamente más conflictivas y menos cohesionadas (Strober, 1981; Johnson \& Flach, 1985 citados en Fornari et al., 1999; Vidovic, et al., 2005)

La evitación de conflictos caracterizaría a las familias anoréxicas. Esta mayor evitación del conflicto por parte de las familias de las adolescentes anoréxicas, trae como consecuencia un aumento en la intensidad, peligrosidad y mantenimiento de la sintomatología, además de incrementar la falta de conciencia de la adolescente acerca de su problema, quien actúa como si su situación fuera normal (González, Hidalgo, Hurtado, Nova \& Venegas, 2002). Rausch y Bay (1997) señalan que las familias evitativas no negocian explícitamente sus diferencias, por lo que quedan sin solución, negando la existencia de problemas y generando la apariencia de "familia ideal", lo que además contribuye a favorecer la falta de conciencia de la adolescente sobre su problemática. Hallazgos del estudio de González et al. (2002), sugieren que a nivel familiar destaca como característica del funcionamiento del sistema, la escasa capacidad de resolución de conflictos familiares. Si la percepción que la adolescente tiene del grado de manejo de situaciones conflictivas, por parte de su familia es negativa, mayor será la susceptibilidad a desarrollar la patología.

Otra variable estudiada por González et al. (2002) corresponde al grado de cohesión familiar. Este estudio encontró que aquellas jóvenes cuyas familias manifestaban una alta cohesión, en términos de apoyo y cercanía emocional, presentaban menor riesgo de padecer un desorden alimentario.

En el mismo sentido, se ha encontrado que ambientes familiares en pacientes con trastorno bulímico han resultado ser menos cohesionados, más conflictivos y menos apoyadores que en familias control. Así mismo, las familias en las cuales se presentan trastornos de alimentación se han percibido a sí mismas como menos apoyadoras, más vulnerables a relaciones conflictuadas y con menos disposición a la expresión de emociones que las familias en las que no hay este tipo de trastornos (Wisotsky, Dancyger, Fornari, Katz, Wisotzky \& Swencionis, 2003).

Por su parte, Waller, Slade y Calam (1990) encontraron que los sujetos con anorexia y bulimia percibían a sus familias con menores niveles de 
adaptabilidad, cohesión y compromiso, en comparación con sujetos normales.

Ordman y Kirschenbaum (1986 citado en Wisotsky et al., 2003) encontraron que pacientes con bulimia que puntuaban más alto que los controles en las subescalas del EDI que miden Tendencia a la Delgadez y Bulimia reportaban menos cohesión y más conflicto en su familia.

\section{Evidencias Contradictorias en Relación con un Patrón Único de Funcionamiento Familiar}

Cook-Darzens et al. (2005) diseñaron un estudio con el propósito de determinar si un patrón específico de funcionamiento familiar autoevaluado se asocia con anorexia nerviosa en adolescentes. Todos los miembros de 40 familias con un adolescente con anorexia nerviosa (padres, madres y hermanos) completaron el instrumento FACES III y los resultados fueron comparados con una muestra de población no clínica. Los resultados tienden a refutar la noción de un patrón psicopatológico específico en estas familias. Hallazgos de este estudio describen la presencia tanto de similitudes como de diferencias entre ambos grupos de familias.

A primera vista, estas tendencias contradicen las teorías tradicionales basadas en modelos de causalidad familiar y discute a favor de una visión más "normativa" de las familias con un miembro con anorexia. También confirma un cuerpo creciente de evidencia que sugiere que las familias de pacientes con anorexia presentan diversos tipos de funcionamiento, probablemente en la gama desde familias sanas en crisis en un extremo, hasta familias muy disfuncionales con alteraciones de muchos años en el otro extremo (Cook-Darzens et al., 2005).

El peso de los factores familiares en la anorexia nerviosa puede, por lo tanto, variar desde una familia a otra, y probablemente dentro de la misma familia a lo largo del tiempo. Uno puede también conjeturar que el episodio de anorexia genera diversas modalidades de adaptación en estas familias más que una sola (Cook-Darzens et al., 2005).

Considerando estos antecedentes teóricos y empíricos, este estudio busca determinar si existe relación entre los síntomas psicológicos asociados al desarrollo de un trastorno de la alimentación en estudiantes secundarias de la comuna de Concepción y el tipo de funcionamiento familiar percibido.

\section{Método}

\section{Tipo de Estudio}

Estudio cuantitativo, mediante un diseño no experimental sobre la base de una encuesta transversal.

\section{Participantes}

La muestra estuvo conformada por 296 alumnas de enseñanza media de la comuna de Concepción, seleccionadas en base a un muestreo no probabilístico por accesibilidad de establecimientos particulares $(n=130,43.9 \%)$, subvencionados $(n=76,25.7 \%)$ y municipalizados $(n=90,30.4 \%)$.

En cuanto a las edades de las participantes, estas variaban de 14 a 19 años, con una media de $16.12(D E=1.22)$. Del total de la muestra, un $26.6 \%$ se encontraba cursando primero medio, un $24.2 \%$ en segundo medio, un $29.0 \%$ en tercero medio y un $20.1 \%$ en cuarto medio.

\section{Instrumentos}

EDI-2. E1 inventario de trastornos alimentarios (EDI-2) es el más extensamente usado y estandarizado de los autoinformes que miden síntomas psicológicos asociados comúnmente a la anorexia nerviosa, bulimia nerviosa y otros trastornos alimentarios. El EDI-2 no proporciona un diagnóstico específico de un desorden alimentario. Se dirige a medir rasgos psicológicos o síntomas que son importantes para entender y tratar los trastornos alimentarios.

El EDI original, introducido en 1983, abarcaba 64 ítems organizados en ocho subescalas. Tres de las subescalas fueron diseñadas para determinar actitudes y comportamientos referentes al comer, al peso y a la forma, estos son, impulso al adelgazamiento, bulimia e insatisfacción con el propio cuerpo, y las cinco restantes, se relacionaban con construcciones más generales de la organización o los rasgos psicológicos clínicos relevantes a los desórdenes alimentarios, como inadaptación, perfeccionismo, desconfianza interpersonal, conocimiento interoceptivo y miedo de la madurez.

La versión actual, el EDI-2, conserva los 64 ítems originales y las ocho subescalas, más tres subescalas adicionales (27 nuevos ítems) que abarcan las subescalas provisionales, es decir, ascetismo, regulación del impulso e inseguridad social. Así, el EDI-2 consiste en 11 subescalas derivadas a partir de 91 ítems frente a los cuales es posible elegir seis alternativas de respuestas que van desde siempre a nunca de acuerdo.

En la presente investigación, se emplearon las tres subescalas del EDI-2 que miden presencia de sintomatología asociada a trastornos de la alimentación. En cuanto a las confiabilidades obtenidas en cada una de ellas, los valores del coeficiente alfa de Cronbach fueron 0.83 para la subescala de Obsesión por la delgadez, 0.71 para la subescala de Bulimia y 0.83 para la subescala de Insatisfacción corporal.

TUSU. El cuestionario Tu-Su, "Cómo es tu familia, cómo es su familia", fue diseñado para evaluar funcionalidad y estructura familiar en los adolescentes y sus familias.

Permite acceder a la percepción de los miembros de la familia respecto a sus propias características de funcionamiento familiar, en relación con su estructura, a los procesos de interacción familiar, eventos estresantes y conductas de riesgos para la salud de los adolescentes y de los adultos.

Este cuestionario se compone de diversas subescalas. Para la presente investigación, se emplearon cuatro de ellas: Comu- 
Tabla 1

Matriz de intercorrelaciones producto-momento de Pearson entre las variables de sintomatología asociada a trastornos de la alimentación y las variables de funcionamiento familiar $(N=296)$

\begin{tabular}{|c|c|c|c|c|c|c|c|}
\hline & 1 & 2 & 3 & 4 & 5 & 6 & 7 \\
\hline 1: Obsesión por Delgadez & $(.83)^{\dagger}$ & & & & & & \\
\hline 2: Bulimia & $.41 * * *$ & $(.71)$ & & & & & \\
\hline 3: Insatisfacción Corporal & $.54 * * *$ & $.46^{* * *}$ & $(.83)$ & & & & \\
\hline 4: Comunicación Madre & -.06 & $-.18 * * *$ & $-.24 * * *$ & $(.87)$ & & & \\
\hline 5: Comunicación Padre & $-.23 * * *$ & $-.28 * * *$ & $-.29 * * *$ & $.47 * * *$ & $(.90)$ & & \\
\hline 6: Cohesión & -.05 & $-.16^{* *}$ & $-.16^{* *}$ & $.59 * * *$ & $.60 * * *$ & $(.75)$ & \\
\hline 7: Manejo Inadecuado de Conflictos & $.12 *$ & $.21 * * *$ & $.19 * * *$ & $-.33^{* * *}$ & $-.35 * * *$ & $-.46 * * *$ & $(.83)$ \\
\hline
\end{tabular}

nicación con la madre, Comunicación con el padre, Cohesión familiar y Manejo inadecuado de conflictos.

$\mathrm{Al}$ analizar las confiabilidades de estas subescalas en base al coeficiente alfa de Cronbach, se obtuvo un valor de 0.87 para la subescala de Comunicación con la madre, 0.90 para la subescala de Comunicación con el padre, 0.75 para la subescala de Cohesión familiar y 0.83 para la subescala de Manejo Inadecuado de Conflictos.

\section{Procedimiento de Obtención de Datos}

La aplicación de los instrumentos se llevó a cabo de modo grupal durante la jornada de clases. En cada sesión de aplicación estaban presentes dos encuestadores debidamente capacitados para dicha tarea, los cuales entregaron las instrucciones para la completación de los cuestionarios y escalas, así como la aclaración de dudas que pudieran surgir.

\section{Estrategia de análisis de datos}

En primer lugar, se realizó un análisis exploratorio de datos a objeto de identificar las características distribucionales de las diversas variables bajo estudio, así como la presencia de valores atípicos. En segundo lugar, se llevó a cabo un análisis descriptivo, con el propósito de obtener los estadísticos de tendencia central y variabilidad de las variables bajo estudio. En tercer lugar, se realizó un análisis bivariado en base al coeficiente de correlación producto-momento de Pearson, a objeto de analizar la relación entre las variables bajo estudio. Finalmente, el análisis de las hipótesis bajo estudio se realizó en base a un análisis de regresión lineal múltiple. Junto a lo anterior, se obtuvieron las confiabilidades de las escalas empleadas mediante el coeficiente alfa de Cronbach.

\section{Resultados}

$\mathrm{Al}$ analizar los puntajes obtenidos en las tres subescalas del EDI-2 que indican presencia de sintomatología asociada a trastornos de la alimentación, se observa que en la subescala de Obsesión por la delgadez la muestra exhibe un promedio de 1.02
$(D E=0.86)$, en la subescala de Bulimia se observa un promedio de $0.37(D E=0.52)$, mientras que en la subescala de Insatisfacción corporal el promedio correspondió a $0.89(D E=0.73)$.

Por su parte, los resultados correspondientes a las subescalas de funcionamiento familiar correspondieron a un promedio de $3.78(D E=0.97)$ para la subescala de Comunicación con la Madre, un promedio de $3.02(D E=1.15)$ para la subescala de Comunicación con el Padre, un promedio de 3.72 $(D E=0.81)$ para la subescala de Cohesión Familiar y un promedio de $1.62(D E=0.50)$ para la subescala de Manejo Inadecuado de Conflictos.

Para llevar a cabo el análisis de las relaciones bivariadas entre las subescalas de sintomatología asociada a trastornos de la alimentación y las subescalas de funcionamiento familiar se empleó el coeficiente de correlación producto-momento de Pearson (véase Tabla 1). Sin embargo, debido a la alta asimetría positiva exhibida por los puntajes obtenidos en las tres subescalas del EDI-2, se llevó a cabo su transformación en base a la obtención de la raíz cuadrada de los puntajes originales. De este modo, todos los resultados que se informan de aquí en adelante se llevan a cabo empleando las variables transformadas.

Como se observa en la Tabla 1, la Obsesión por la Delgadez muestra relaciones significativas sólo con dos de las cuatro variables de funcionamiento familiar analizadas: Comunicación con el Padre $(r(294)=-0.23, p<0.001)$ y Manejo Inadecuado de Conflictos $(r(294)=0.12, p<0.05)$.

Por su parte, tanto Bulimia como Insatisfacción Corporal muestran un patrón similar de correlaciones con el conjunto de variables de funciona- 
Tabla 2

Coeficientes, niveles de significación y correlaciones semiparciales al cuadrado para los resultados de la regresión lineal múltiple sobre los puntajes de la variable Obsesión por la Delgadez $(N=296)$

\begin{tabular}{|c|c|c|c|c|c|}
\hline & \multicolumn{2}{|c|}{$\begin{array}{l}\text { Coeficientes no } \\
\text { estandarizados }\end{array}$} & \multirow{2}{*}{$\begin{array}{c}\begin{array}{c}\text { Coeficientes } \\
\text { estandarizados }\end{array} \\
\beta\end{array}$} & \multirow[t]{2}{*}{$t$} & \multirow[t]{2}{*}{$s r^{2}$} \\
\hline & $B$ & $E E$ & & & \\
\hline (constante) & .70 & .23 & & $3.04 *$ & \\
\hline Comunicación Madre & .01 & .04 & .02 & .33 & .00 \\
\hline Comunicación Padre & -.14 & .03 & -.30 & $-4.20 * * *$ & .06 \\
\hline Cohesión & .10 & .05 & .16 & $1.91 *$ & .01 \\
\hline Manejo Inadecuado de Conflictos & .10 & .07 & .09 & $1.44 *$ & .00 \\
\hline
\end{tabular}

$*: p<0.05 ; * *: p<0.01 ; * * *: p<0.001$

miento familiar. Para el caso de Bulimia se tienen relaciones significativas con: Comunicación con la Madre $(r(294)=-0.18, p<0.001)$, Comunicación con el Padre $(r(294)=-0.28, p<0.001)$, Cohesión $(r(294)=-0.16, p<0.01)$ y Manejo Inadecuado de Conflictos $(r(294)=0.21, p<0.001)$. En el caso de Insatisfacción Corporal, las correlaciones significativas corresponden a: Comunicación con la Madre $(r(294)=-0.24, p<0.001)$, Comunicación con el Padre $(r(294)=-0.29, p<0.001)$, Cohesión $(r(294)=$ $-0.16, \mathrm{p}<0.01)$ y Manejo Inadecuado de Conflictos $(r(294)=0.19, p<0.001)$.

Para evaluar el efecto del conjunto de variables de funcionamiento familiar sobre cada una de las tres variables de sintomatología asociada a trastornos de la alimentación, se llevaron a cabo regresiones lineales múltiples. En cada una de estas regresiones, la variable criterio correspondió a la transformación mediante raíz cuadrada de cada una de las variables de sintomatología asociada a trastornos de la alimentación. Al replicar estos análisis con las variables criterio original (sin transformar), se observaron problemas en el cumplimiento de los supuestos de normalidad en la distribución de los residuos, así como presencia de heterocedasticidad de los mismos. Dichos problemas disminuyeron de manera importante al trabajar con las variables criterio transformadas mediante raíz cuadrada. Esto justifica la elección de estas últimas como las variables a analizar en el presente estudio.

La primera regresión empleó como variable criterio la Obsesión por la Delgadez y como predictores las cuatro variables de características del funcionamiento familiar. El resultado obtenido correspondió a un coeficiente $R^{2}=0.07\left(R_{a d j}^{2}=0.06\right)$. En cuanto a la significación estadística de este modelo, el resultado mostró un $F(4,291)=5.56(p<0.001)$ lo cual permite sostener que existe una relación li- neal significativa entre los predictores y los puntaje correspondientes a la Obsesión por la Delgadez. Los valores de los coeficientes para el modelo se presentan en la Tabla 2.

Del análisis de la Tabla 2 se puede concluir que sólo dos de los cuatro predictores resultan ser estadísticamente significativos en su contribución a la explicación de los puntajes de la variable Obsesión por la Delgadez. El primero de ellos corresponde a Comunicación con el Padre que exhibe una relación inversa con la Obsesión por la Delgadez $(\beta=-0.30$, $\left.s r^{2}=0.06\right)$, mientras que el segundo corresponde a la Cohesión Familiar $\left(\beta=0.16, s r^{2}=0.01\right)$ que exhibe una relación directa con la variable criterio. Sin relación estadísticamente significativa aparecen Comunicación con la Madre $(p>0.05)$ y Manejo Inadecuado de Conflictos $(p>0.05)$.

La segunda regresión empleó como variable criterio los puntajes de Bulimia. El resultado obtenido correspondió a un coeficiente $R^{2}=0.10$ $\left(R_{a d j}^{2}=0.09\right)$. En cuanto a la significación estadística de este modelo, el resultado mostró un $F(4,291)=8.17(p$ $<0.001)$ lo cual permite sostener que existe una relación lineal significativa entre el conjunto de predictores y la variable criterio. Los valores de los coeficientes para el modelo se presentan en la Tabla 3.

Como se aprecia en la Tabla 3, Bulimia muestra una relación inversa y significativa con la variable Comunicación con el Padre $\left(\beta=-0.26, s r^{2}=0.04\right)$ $\mathrm{y}$ una relación directa y significativa con Manejo Inadecuado de Conflictos $\left(\beta=0.15, s r^{2}=0.02\right)$. Sin relación estadísticamente significativa aparecen Comunicación con la Madre $(p>0.05)$ y Cohesión $(p>0.05)$.

Finalmente, al llevar a cabo el análisis de regresión sobre los puntajes de la variable Insatisfacción Corporal, se obtiene un coeficiente $R^{2}=0.11\left(R_{a d j}^{2}\right.$ $=0.10)$. En cuanto a la significación estadística de 
Tabla 3

Coeficientes, niveles de significación y correlaciones semiparciales al cuadrado para los resultados de la regresión lineal múltiple sobre los puntajes de la variable Bulimia $(N=296)$

\begin{tabular}{|c|c|c|c|c|c|}
\hline & \multicolumn{2}{|c|}{ Coeficientes no estandarizados } & \multirow{2}{*}{$\begin{array}{c}\text { Coeficientes estandarizados } \\
\beta \\
\end{array}$} & \multirow{2}{*}{$\mathrm{t}$} & \multirow{2}{*}{$\mathrm{sr}^{2}$} \\
\hline & $B$ & $E E$ & & & \\
\hline (constante) & .42 & .19 & & $2.16^{*}$ & \\
\hline Comunicación Madre & -.03 & .03 & -.07 & -.94 & .00 \\
\hline Comunicación Padre & -.10 & .03 & -.26 & $-3.71 * * *$ & .04 \\
\hline Cohesión & .06 & .04 & .10 & 1.29 & .01 \\
\hline Manejo Inadecuado de Conflictos & .13 & .06 & .15 & $2.35^{*}$ & .02 \\
\hline
\end{tabular}

$*: p<0.05 ; * *: p<0.01 ; * * *: p<0.001$

Tabla 4

Coeficientes, niveles de significación y correlaciones semiparciales al cuadrado para los resultados de la regresión lineal múltiple sobre los puntajes de la variable Insatisfacción Corporal $(N=296)$

\begin{tabular}{|c|c|c|c|c|c|}
\hline & \multicolumn{2}{|c|}{ Coeficientes no estandarizados } & \multirow{2}{*}{$\frac{\text { Coeficientes estandarizados }}{\beta}$} & \multirow{2}{*}{$\mathrm{t}$} & \multirow{2}{*}{$\mathrm{sr}^{2}$} \\
\hline & $B$ & $E E$ & & & \\
\hline (constante) & .98 & .18 & & $5.32 * * *$ & \\
\hline Comunicación Madre & -.07 & .03 & -.17 & $-2.41^{*}$ & .02 \\
\hline Comunicación Padre & -.09 & .03 & -.25 & $-3.57 * * *$ & .04 \\
\hline Cohesión & .07 & .04 & .14 & 1.75 & .01 \\
\hline Manejo Inadecuado de Conflictos & .09 & .05 & .11 & 1.74 & .01 \\
\hline
\end{tabular}

$*: p<0.05 ; * *: p<0.01 ; * * *: p<0.001$

este modelo, el resultado mostró un $F(4,291)=$ $9.04(p<0.001)$ lo cual permite sostener que existe una relación lineal significativa entre el conjunto de predictores y los puntajes de la variable Insatisfacción Corporal. Los valores de los coeficientes para el modelo se presentan en la Tabla 4.

Los resultados de este análisis (véase Tabla 4) ponen en evidencia que, tanto Comunicación con la Madre $\left(\beta=-0.17, s r^{2}=0.02\right)$, como Comunicación con el Padre $\left(\beta=-0.25, s r^{2}=0.04\right)$ presentan relaciones inversas y significativas con la variable Insatisfacción Corporal.

\section{Discusión}

Uno de los principales hallazgos de este estudio mostró que la Obsesión por la Delgadez, es decir, la preocupación excesiva por las dietas, la preocupación por el peso y el miedo al aumento del peso muestra relaciones significativas con dos de los cuatro predictores analizados: Comunicación con el Padre y Manejo Inadecuado de Conflictos. Tal como señalara tempranamente Salvador Minuchin et al. (1978), la evitación de conflictos sería una de las características predominantes de interacción que mantienen la "somatización". La evitación de conflictos caracterizaría a las familias anoréxicas. González et al. (2002) señalan que esta evitación trae como consecuencia un aumento en la intensidad, peligrosidad y mantenimiento de la sintomatología, además de incrementar la falta de conciencia de la adolescente acerca de su problema, quien actúa como si su situación fuera normal. Lo anterior también lo afirman Rausch y Bay (1997) quienes señalan que las familias evitativas, no negocian explícitamente sus diferencias, por lo que quedan sin solución, negando la existencia de problemas y generando la apariencia de "familia ideal", lo que además contribuye a favorecer la falta de conciencia de la adolescente sobre su problemática.

Por su parte, tanto Bulimia, es decir, la tendencia a pensar y a realizar conductas relacionadas con episodios de ingesta excesiva de alimentos, como Insatisfacción Corporal, o sea, el descontento con la forma total y la preocupación extraordinaria del tamaño de algunas regiones del cuerpo (estómago, 
caderas, muslos, nalgas) muestran un patrón similar de correlaciones con el conjunto de predictores. Para el caso de Bulimia se tienen relaciones significativas con: Comunicación con la Madre, Comunicación con el Padre, Cohesión y Manejo Inadecuado de Conflictos. En el caso de Insatisfacción Corporal, las correlaciones significativas corresponden a: Comunicación con la Madre, Comunicación con el Padre, Cohesión y Manejo Inadecuado de Conflictos. Cabe destacar que la insatisfacción corporal ha sido conceptualizada como uno de los aspectos de la "distorsión de la imagen corporal" característica de los trastornos alimentarios. Este hallazgo coincide con Leung, Schwartzman y Steiger (1996) quienes encontraron que las familias disfuncionales, con bajos niveles de adaptabilidad y cohesión, predicen elevados puntajes en la subescala del EDI de Insatisfacción Corporal en una población no clínica y puede contribuir al desarrollo del trastorno.

Se puede concluir que sólo dos de los cuatro predictores resultan ser estadísticamente significativos en su contribución a la explicación de los puntajes de la variable Obsesión por la Delgadez. El primero de ellos corresponde a Comunicación con el Padre que exhibe una relación inversa. Podríamos pensar, entonces que los progenitores varones, que en general han sido dejados de lado de las investigaciones en trastornos de alimentación, podrían estar jugando un rol fundamental. Este parece ser uno de los hallazgos más relevantes de esta investigación, ya que en términos preventivos, los padres debieran ser incorporados activamente con el fin de evitar la aparición de un trastorno alimentario.

El segundo corresponde a la Cohesión Familiar que exhibe una relación directa con la variable criterio. Esto va en la misma línea de estudios previos que describen relaciones entre el nivel de cohesión familiar y la presencia de trastornos de la alimentación.

Por otra parte, Bulimia muestra una relación inversa y significativa con la variable Comunicación con el Padre y una relación directa y significativa con Manejo Inadecuado de Conflictos.

Existe una relación lineal significativa entre el conjunto de predictores y los puntajes de la variable Insatisfacción Corporal. Tanto Comunicación con la Madre como Comunicación con el Padre presentan relaciones inversas y significativas con la variable Insatisfacción Corporal.

Parece relevante destacar que la relación esperada entre el funcionamiento familiar percibido y los trastornos de la alimentación en pacientes, se repite en población no clínica, que es la estudiada en esta investigación. En ese sentido, las implicancias a nivel práctico de estos resultados, se relacionarían con aspectos preventivos. Ante estos hallazgos parece fundamental incorporar a la familia en programas de prevención de trastornos de la alimentación, que favorezcan la comunicación con ambos padres, que permitan un ambiente familiar menos cohesionado y que facilite la resolución adecuada de los conflictos.

Siguiendo lo anterior, habría sido interesante contar con la percepción de funcionamiento familiar de los padres, pero el nivel de colaboración de éstos, no permitió establecer resultados. Sería relevante establecer comparaciones entre ambas percepciones (adolescentes versus padres), ya que si los progenitores no perciben disfuncionalidad entonces difícilmente participarán en programas de prevención, por lo que sería relevante evaluar estrategias para fomentar la colaboración de los padres.

Parece importante recalcar, que si queremos intervenir preventivamente en los Trastornos Alimentarios, el trabajo tendrá que estar orientado a los factores individuales y sociofamiliares, que generan vulnerabilidad ya que estos serán los que marcarán en la adolescencia, el tipo de respuesta entendida como conducta de riesgo que los jóvenes asumirán. Considerando la importancia de la familia en la génesis y mantenimiento de los trastornos de la alimentación, es importante considerar dentro de las estrategias preventivas la discusión y confrontación con habilidades de resolución de problemas para conseguir soluciones constructivas frente a las dificultades de relación entre padres e hijos.

Ahora bien, se podría hipotetizar que las adolescentes pudieran tener una peor percepción de su funcionamiento familiar dada la etapa del ciclo evolutivo en que se encuentran. Están en pleno proceso de consolidación de su identidad, por lo que se produce un distanciamiento de la familia y un acercamiento a su grupo de pares. Lo anterior concuerda con los señalado por Dancyger, et al. (2005) quienes encontraron diferencias estadísticamente significativas entre las pacientes y sus padres. Las madres consideraban que el funcionamiento familiar era saludable y poco caótico, en cambio, sus hijas no coincidieron con tal reporte.

Pero por otra parte, si la percepción de mal funcionamiento de parte de las jóvenes juega un rol fundamental en la presencia del trastorno, entonces, independiente de la percepción de sus padres, habría que intervenir preventivamente. 
La presente investigación tiene una limitación que es característica dentro de los estudios en trastornos de la alimentación, esto es, que es correlacional, lo que pone dificultades en la determinación de si la disfuncionalidad familiar contribuye al desarrollo de sintomatología relativa a trastornos de la alimentación o si es el trastorno el que contribuye a la disfunción familiar.

Otra limitación de este estudio es el tipo de muestra, es decir, no hay aleatorización de las participantes. Y por otra parte, se debe ser cuidadoso al establecer conclusiones con medidas de autorreporte, ya que ambos instrumentos tienen esa característica y, de alguna manera, las respuestas podrían estar teñidas por la deseabilidad social. Por lo tanto, se debe hacer hincapié en la característica "percibido" del funcionamiento familiar.

Finalmente, como sugerencia para futuras investigaciones sería interesante incorporar muestra clínica como una forma de corroborar los hallazgos de este estudio, y poder comparar ambos grupos. Además, es relevante incorporar la percepción de los padres a través de medidas de autorreporte, pero además observaciones clínicas que permitan contrastar información.

\section{Referencias}

American Psychiatric Association (APA) (2002). DSM IV-TR. Manual diagnóstico y estadístico de los trastornos mentales: Texto revisado. Barcelona: Masson.

Behar, R. (2004). Trastornos de la conducta alimentaria: Clínica y epidemiología. En R. Behar \& G. Figueroa (Eds.), Anorexia nerviosa y bulimia. Clínica y terapéutica (pp. 17-53). Santiago: Mediterráneo.

Carrasco, E. (2004). Perspectiva familiar en el tratamiento de los trastornos de la conducta alimentaria. En R. Behar \& G. Figueroa (Eds.), Anorexia nerviosa y bulimia. Clínica y terapéutica (pp. 235-257). Santiago: Mediterráneo.

Cook-Darzens, S., Doyen, C., Falissard, B. \& Mouren, M. C. (2005). Self-perceived family functioning in 40 French families of anorexic adolescents: Implications for therapy. European Eating Disorders Review, 13, 223-236.

Dancyger, I., Fornari, V., Scionti, L., Wisotsky, W. \& Sunday, S. (2005). Do daughters with eating disorders agree with their parents' perception of family functioning? Comprehensive Psychiatry, 46, 135-139.

Fornari, V., Wlodarczyk-Bisaga, K., Matthews, M., Sandberg, D., Mandel, F. \& Katz, J. L. (1999). Perception of family functioning and depressive symptomatology in individuals with anorexia nervosa or bulimia nervosa. Comprehensive Psychiatry. 40, 434-441.

García-Palacios, A., Rivero, I. \& Botella, C. (2004). Personalidad y trastornos de la conducta alimentaria. Comparación entre una muestra control y una muestra desde un enfoque categorial y dimensional. Revista Argentina de Clínica Psicológica, 2,91-109.
González, L., Hidalgo, M., Hurtado, M., Nova, C. \& Venegas, M. (2002). Relación entre factores individuales y familiares de riesgo para desórdenes alimenticios en alumnos de enseñanza media. Revista de Psicología de la Universidad de Chile, 11, 91-115.

Hernández, M. (2006). Las influencias familiar y social en la anorexia y la bulimia, el caso de Saltillo, México. Episteme, 8 y 9. Recuperado el 14 de marzo de 2007 desde: http://www. uvmnet.edu/investigacion/episteme/numero8y9-06/reportes/ a_anorexia.asp

Hoek, H. \& Van Hoeken, D. (2003). Review of the prevalence and incidence of eating disorders. Internacional Journal of Eating Disorders, 34, 383-396.

Hoeken, D., Seidell, J. \& Hoek, H. (2003). Epidemiology. En J. Treasure, U. Schmidt \& E. van Furth (Eds.), Handbook of eating disorders. (2da. Ed.) (pp. 11-34). West Sussex: Wiley \& Sons.

Leung, F., Schwartzman, A. \& Steiger, H. (1996). Testing a dual-process family model in understanding the development of eating pathology: A structural equation modeling analysis. International Journal of Eating Disorders, 20, 367-375.

Marín, V. (2002). Trastornos de la conducta alimentaria en escolares y adolescentes. Revista Chilena de Nutrición, 2, 86-91.

Maturana, A. (2003). Trastornos de la conducta alimentaria en niños y adolescentes. En C. Almonte, M. E. Montt \& A. Correa (Eds.), Psicopatología infantil y de la adolescencia. Santiago: Mediterráneo.

Olson, D. H., Portner, J. \& Bell, R. Q. (1982). FACES II: Family Adaptability and Cohesion Evaluation Scales. Minnesota: Family Social Science, University of Minnesota.

Perpiñá, C. (1995). Trastornos alimentarios. En A. Belloch, F. Ramos \& B. Sandín (Eds.), Manual de psicopatología Vol. 1 (pp. 531-557). Madrid: McGraw-Hill.

Pompili, M., Mancinelli, I., Girardi, P., Ruberto, A. \& Tatarelli, R. (2004). Suicide in anorexia nervosa: A meta-analysis. Internacional Journal of Eating Disorders, 36, 99-103.

Rausch, C. \& Bay, L. (1997). Anorexia nerviosa y bulimia: Amenazas a la autonomía ( $1^{\mathrm{a}}$ Ed.). Buenos Aires: Paidós.

Sarason, B. \& Sarason, I. (1996). Conductas inadaptadas en la niñez y adolescencia. En B. Sarason \& I. Sarason (Eds.), Psicología anormal: El problema de la conducta inadaptada. (pp. 484-490). México: Prentice Hall.

Steinhausen, H., Boyadjieva, S., Grigoroiu-Serbanescu, M., Seidel, R. \& Winkler Metzke, C. (2000). A transcultural outcome study of adolescent eating disorders. Acta Psychiatrica Scandicavica, 101, 60-66.

Vidovic, V., Juresa, V., Begovac, I., Mahnik, M. \& Tocilj, G. (2005). Perceived family cohesion, adaptability and communication in eating disorders. European Eating Disorders Review, 13, 19-28.

Vitousek, K. \& Manke, F. (1994). Personality variables and disorders in anorexia nervosa and bulimia nervosa. Journal of Abnormal Psychology, 103, 137-147.

Waller, G., Calam, R. \& Slade, P. (1988). Family interaction and eating disorders: Do family members agree? British Review of Bulimia and Anorexia, 3, 33-40.

Waller, G., Slade, P. \& Calam, R. (1990). Family adaptability and cohesion: Relation to eating attitudes and disorders. International Journal of Eating Disorders, 9, 225-228.

Wisotsky, W., Dancyger, I., Fornari, V., Katz, J., Wisotzky, W. \& Swencionis, C. (2003). The relationship between eating pathology and perceived family functioning in eating disorder patients in a day treatment program. Eating Disorders, 11, 89-99. 